\title{
Copper Oxide Nanoparticles Induce Oxidative DNA Damage and Cell Death via Copper lon-Mediated P38 MAPK Activation in Vascular Endothelial Cells
}

This article was published in the following Dove Press journal:

International Journal of Nanomedicine

\author{
Hui He ${ }^{l, *}$ \\ Zhen Zou (iD) ${ }^{2, *}$ \\ Bin Wang iD $^{2}$ \\ Ge $X u^{2}$ \\ Chengzhi Chen (iD) ${ }^{3}$ \\ Xia Qin' \\ Chao Yu' \\ Jun Zhang iD $^{2}$ \\ 'College of Pharmacy, Chongqing Medical \\ University, Chongqing, People's Republic \\ of China; ${ }^{2}$ Institute of Life Sciences, \\ Chongqing Medical University, \\ Chongqing, People's Republic of China; \\ ${ }^{3}$ School of Public Health and \\ Management, Chongqing Medical \\ University, Chongqing, People's Republic \\ of China \\ *These authors contributed equally to \\ this work
}

Background: Inhaled nanoparticles can cross pulmonary air-blood barrier into circulation and cause vascular endothelial injury and progression of cardiovascular disease. However, the molecular mechanism underlying the vascular toxicity of copper oxide nanoparticles (CuONPs) remains unclear. We have recently demonstrated that the release of copper ions and the accumulation of superoxide anions contributed to CuONPs-induced cell death in human umbilical vein endothelial cells (HUVECs). Herein, we further demonstrate the mechanism underlying copper ions-induced cell death in HUVECs.

Methods and Results: CuONPs were suspended in culture medium and vigorously vortexed for several seconds before exposure. After treatment with CuONPs, HUVECs were collected, and cell function assays were conducted to elucidate cellular processes including cell viability, oxidative stress, DNA damage and cell signaling pathways. We demonstrated that CuONPs uptake induced DNA damage in HUVECs as evidenced by $\gamma \mathrm{H} 2 \mathrm{AX}$ foci formation and increased phosphorylation levels of ATR, ATM, p53 and H2AX. Meanwhile, we showed that CuONPs exposure induced oxidative stress, indicated by the increase of cellular levels of superoxide anions, the upregulation of protein levels of heme oxygenase-1 (HO-1) and glutamate-cysteine ligase modifier subunit (GCLM), the elevation of the levels of malondialdehyde (MDA), but the reduction of glutathione to glutathione disulfide ratio. We also found that antioxidant $\mathrm{N}$-acetylL-cysteine (NAC) could ameliorate CuONPs-induced oxidative stress and cell death. Interestingly, we demonstrated that p38 mitogen-activated protein kinase (MAPK) signaling pathway was activated in CuONPs-treated HUVECs, while p38 $\alpha$ MAPK knockdown by siRNA significantly rescued HUVECs from CuONPs-induced DNA damage and cell death. Importantly, we showed that copper ions chelator tetrathiomolybdate (TTM) could alleviate CuONPs-induced oxidative stress, DNA damage, p38 MAPK pathway activation and cell death in HUVECs.

Conclusion: We demonstrated that CuONPs induced oxidative DNA damage and cell death via copper ions-mediated p38 MAPK activation in HUVECs, suggesting that the release of copper ions was the upstream activator for CuONPs-induced vascular endothelial toxicity, and the copper ions chelator TTM can alleviate CuONPs-associated cardiovascular disease. Keywords: CuONPs, vascular endothelial cell death, oxidative stress, DNA damage, p38 MAPK activation, copper ions release

\section{Introduction}

Copper oxide nanoparticles (CuONPs) is important metal oxide nanomaterial, which exhibits good conductive, catalytic and antibacterial properties. It has been widely used
Correspondence: Chao Yu; Jun Zhang Email yuchaom@163.com;

zhangjun@cqmu.edu.cn
International Journal of Nanomedicine 2020:15 329|-3302

3291

DovePress $f$ in $\boldsymbol{v}$

http://doi.org/10.21471/N.S241157 (c) (1) (8) 2020 He et al. This work is published and licensed by Dove Medical Press Limited. The full terms of this license are avalable at https://www.dovepress.com/terms.php (c) you hereby accept the Terms. Non-commercial uses of the work are permitted without any further permission from Dove Medical Press Limited, provided the work is properly attributed. For permission for commercial use of this work, please see paragraphs 4.2 and 5 of our Terms (https://www.dovepress.com/terms.php). 
as semiconductors, ${ }^{1}$ oxidation catalysts, ${ }^{2}$ energy storage materials, ${ }^{3}$ and antibacterial agents. ${ }^{4}$ Despite its widespread industrial and biomedical application, concerns regarding potential toxic impacts of CuONPs have increased rapidly. CuONPs have been reported as the most toxic metal oxide nanoparticles. ${ }^{5,6}$ Previous in vitro studies have reported that CuONPs can induce cytotoxicity in numerous cell types, including human A549 lung epithelial cells, ${ }^{7-9}$ MCF7 breast cancer cells, ${ }^{10}$ HL6 0promyelocytic leukemic cells, ${ }^{11} \mathrm{HepG} 2$ liver hepatocellular carcinoma cells, ${ }^{12}$ frog A6 kidney epithelial cells ${ }^{13}$ and rat primary brain astrocytes. ${ }^{14}$ Still, the molecular mechanism underlying CuONPs cytotoxicity remains incompletely understood.

Cardiovascular disease is the number one cause of death globally. ${ }^{15}$ Vascular endothelial cells form the inner surface of blood vessels, whose dysfunction leads to the development of cardiovascular disease. ${ }^{16}$ Increasing evidence has indicated that engineered nanoparticles exposure may penetrate pulmonary air-blood barrier into circulation. Recently, Miller et al, further clarified that inhaled nanoparticles selectively accumulated at sites of vascular inflammation in both animal models of disease and in human volunteers, ${ }^{17}$ suggesting that inhaled nanoparticles can directly interact with vascular endothelial cell, resulting in endothelial injury and acceleration of cardiovascular diseases.

It has been suggested that the toxicity of metal oxide nanoparticles results from the release of metal ions or/and the nanoparticles themselves. Our recent study demonstrated that autophagy-dependent release of zinc ions was involved in human A549 lung epithelial cell death and acute lung injury in mice induced by zinc oxide nanoparticles exposure. ${ }^{18,19}$ Hanagata et al reported that the released copper ions, rather than CuONPs themselves, mediated CuONPs toxicity in A549 lung epithelial cells. ${ }^{9}$ Recently, we also demonstrated that lysosomal deposition of CuONPs facilitated copper ions release and triggered CuONPs-induced human umbilical vein endothelial cells (HUVECs) death. ${ }^{20}$ However, the exact roles of copper ions in CuONPs toxicity need further be investigated.

Reactive oxygen species (ROS) are chemically reactive molecules, which mainly originate from damaged mitochondria. ROS are mainly composed of superoxide anions $\left(\mathrm{O}_{2}{ }^{-}\right)$, hydrogen peroxide $\left(\mathrm{H}_{2} \mathrm{O}_{2}\right)$ and hydroxyl free radical $(\cdot \mathrm{OH})$. Oxidative stress, indicated by increased intracellular ROS, is associated with nanoparticles cytotoxicity. ${ }^{21}$ Oxidative stress can result in oxidized nucleic acids, amino acids, carbohydrates and lipids. ${ }^{22}$ Previous studies have suggested that nanoparticles-induced oxidation of nucleic acids could cause DNA damage, cell cycle arrest and subsequent cell death. ${ }^{23,24}$ Our recent study also demonstrated that CuONPs exposure caused mitochondrial dysfunction and the accumulation of mitochondrial superoxide anions in HUVECs. ${ }^{25}$

In the current study, we clarified the potential links between the release of copper ions, oxidative stress response and $\mathrm{p} 38$ MAPK activation in CuONPs-induced cytotoxicity in vascular endothelial cells. We demonstrated that the copper ions released from CuONPs induced oxidative stress and p38 MAPK signaling activation, which subsequently caused DNA damage and cell death in HUVECs. Our findings contribute to clarifying the mechanisms underlying CuONPs toxicity in the circulation system and suggest that the chelation of copper ions may be an effective means of alleviating CuONPs toxicity.

\section{Materials and Methods Materials and Cell Culture}

CuONPs $(<50 \mathrm{~nm}$ particle size, \#544868) were purchased from Sigma Aldrich (St. Louis, MO, USA) and characterized as previously described. ${ }^{20}$ Human umbilical vein endothelial cells (HUVECs), the most widespread in vitro model for studying endothelial physiology and pathology, was obtained from the American Type Culture Collection (Rockville, MD, USA) and was cultured in Dulbecco's modified Eagle's medium (DMEM, Thermo Fisher Scientific, Waltham, MA, USA) supplemented with $10 \%$ fetal bovine serum and $100 \mathrm{U}$ penicillinstreptomycin at $37^{\circ} \mathrm{C}$ in a $5 \% \mathrm{CO}_{2}$ humidified incubator.

\section{Transmission Electron Microscopy}

Transmission electron microscopy (TEM) was used to characterize CuONPs morphology. Briefly, a few drops of CuONPs solution in deionized water were added to carboncoated copper electron microscope grids and observed under a TEM (Hitachi-7500, Japan) after drying at room temperature. Cell ultrastructure was also observed under TEM. After treatment and subsequent trypsinization, cells were centrifuged at $1200 \mathrm{rpm} / \mathrm{min}$ for $5 \mathrm{~min}$ and fixed using $4 \%$ glutaraldehyde for $2 \mathrm{~h}$ at $4^{\circ} \mathrm{C}$. Then, cells were postfixed using $1 \%$ osmium tetroxide for $1 \mathrm{~h}$ at $4^{\circ} \mathrm{C}$, dehydrated in a graded series of alcohol and acetone and embedded in Epon 816 (Electron Microscopy Sciences, Hatfield, PA, USA). Ultrathin sections were prepared using a Leica ultramicrotome (Leica Microsystems, Buffalo Grove, IL, USA) and stained with uranyl acetate-lead citrate. TEM images were obtained using a JEM-1400Plus transmission electron microscope (JEOL Ltd. Tokyo, Japan). 


\section{MTS Cell Viability Assay}

Cell viability was assayed using CellTiter $96^{\circledR}$ AQueous One Solution Cell Proliferation Assay (MTS) kit (\#G3581, Promega, Madison, WI, USA) as described previously. ${ }^{19}$ Briefly, HUVECs were seeded in 96-well plates overnight. After treatment with CuONPs or other reagents for indicated times, the MTS solution was diluted 10-fold in DMEM and subsequently added to each well of the cultured plate. The plate was incubated at $37^{\circ} \mathrm{C}$ for $1 \mathrm{~h}$ and the absorbance of each well at $490 \mathrm{~nm}$ was measured using a VERS Amax Microplate Reader (Molecular Devices Corp, Sunnyvale, CA, USA). All assays were conducted in triplicate.

\section{Fluorescence Activated Cell Sorting (FACS) Assay}

FACS assays were performed as previously described. ${ }^{20,25,26}$ Calcein AM (50 nM, Thermo Fisher Scientific) was used to determine cell viability. DHE (5 nM, Beyotime, Jiangsu, China) and MitoSOX $(5 \mu \mathrm{M}$, Thermo Fisher Scientific) were used to measure cellular superoxide anions and mitochondrial superoxide anions, respectively. All FACS assays were performed using CytoFLEX Platform (Beckman Coulter, Miami, FL, USA), and the results were analyzed using FlowJo Version VX software (TreeStar, Ashland, OR, USA). All assays were conducted in triplicate.

\section{Comet Tail Assay}

Comet tail assay was conducted as described previously. ${ }^{27}$ Briefly, HUVECs seeded in 12-well plates were treated using CuONPs $(20 \mu \mathrm{g} / \mathrm{mL})$ for $12 \mathrm{~h}$. Then, the cells were collected and added a few droplets of low-melting agarose (Amresco, Solon, USA). The cell mixture was then spread onto a slide precoated with normal-melting agarose (Amresco, Solon, OH, USA), and incubated in lysis buffer at $4^{\circ} \mathrm{C}$ for $1 \mathrm{~h}$. Then, the slides were placed in electrophoresis buffer for $30 \mathrm{~min}$ and neutralized in water. After dehydration at room temperature, the slides were stained using ethidium bromide (Amresco, Solon, OH, USA) and observed under a fluorescence microscope (Eclipse TiU, Nikon Corp., Tokyo, Japan).

\section{Immunofluorescence Staining}

Cells were fixed using cold methanol for $15 \mathrm{~min}$ and subsequently permeabilized with $0.2 \%$ Triton X-100 in phosphate buffer saline (PBS) for $10 \mathrm{~min}$ at room temperature. After blocking with $2 \%$ bovine serum albumin in PBS for $1 \mathrm{~h}$ at room temperature, cells were incubated with a primary antibody against phospho-histone H2AX (S139) $(\gamma \mathrm{H} 2 \mathrm{AX}, 1: 100$,
Cell Signaling Technology, \#9508) diluted in a blocking solution at room temperature for $1 \mathrm{~h}$. After three washes with PBS, the cells were incubated with Alexa Fluor 488-conjugated donkey anti-rabbit IgG secondary antibody was used (1:500, Molecular Probes, \#A-21207) and 4',6-diamidino-2-phenylindole (DAPI, Molecular Probes, Eugene, OR, USA) diluted in PBS for $1 \mathrm{~h}$ at room temperature. After washing with PBS, the coverslips were sealed using nail polish. Fluorescence images were obtained using a Nikon A1R+/A1 Confocal Microscope (Nikon, Tokyo, Japan).

\section{Western Blotting Assay}

Cells were directly lysed using $2 \times$ protein lysing buffer $(2 \%$ SDS, $5 \% \beta$-mercaptoethanol, $0.5 \%$ sucrose and $0.2 \%$ bromophenol blue) at $4^{\circ} \mathrm{C}$ for $20 \mathrm{~min}$, and then the lysates were heated in a metal bath at $98^{\circ} \mathrm{C}$ for $5 \mathrm{~min}$. Cell lysates were separated on 4-12\% SDS-PAGE gels and transferred to polyvinylidene fluoride (PVDF) membranes (Merck Millipore, Billerica, MA, USA). The membranes were blocked using 5\% non-fat milk and immunoblotted using indicated antibodies. The protein signals were detected using a New-SUPER ECL Substrate Kit (Keygen Biotech, Nanjing, China). The primary antibodies used in this study targeted phospho-ATR (S428) (1:1000, abcam, ab178407), phospho-ATM (S1981) (1:1000, Cell Signaling Technology, \#5883), phospho-p53 (S15) (1:1000, abcam, ab223868), phospho-histone H2AX (S139) (1:1000, Cell Signaling Technology, \#9508), GCLM (1:1000, abcam, ab126704), HO-1 (1:1000, abcam, ab68477), phospho-p38 (T180/Y182) (1:500, Santa Cruz Biotechnology, sc-17852-R), phospho-Hsp27 (S82) (1:1000, abcam, ab155987), phospho-ATF2 (T71) (1:1000, abcam, ab32019) and Actin (1:3000, Bioss, \#bs-0061R). The secondary antibodies used in this study were horseradish peroxidase (HRP)conjugated goat anti-mouse IgG $(1: 10,000$, TransGen Biotech, Beijing, China, \#HS201-01) and HRP-conjugated goat antirabbit $\operatorname{IgG}(1: 10,000$, TransGen Biotech, Beijing, China, \#HS101-01).

\section{GSH/GSSG Assay}

Reduced (GSH) and oxidized (GSSG) glutathione in CuONPs-treated HUVECs were measured spectrophotometrically using a GSH and GSSG Assay Kit (Beyotime, Shanghai, China). Briefly, HUVECs were added in a threefold volume of protein removal reagent $\mathrm{M}$ and fully vortexed. Then, the cell samples were freeze-thawed in liquid nitrogen and a $37^{\circ} \mathrm{C}$ water bath. After centrifuging at $10,000 \mathrm{~g}$ for 10 $\min$ at $4{ }^{\circ} \mathrm{C}$, the supernatant was used for the determination of 
the GSH/GSSG ratio in accordance with the manufacturer's protocol. All assays were conducted in triplicate.

\section{MDA Assays}

The level of MDA in the CuONPs-treated HUVECs was assayed using a Lipid Peroxidation MDA Assay Kit (Beyotime, Shanghai, China). Briefly, HUVECs seeded into 12-well plates were directly lysed using cell lysis buffer (Beyotime, Shanghai, China). The lysate was centrifuged for $10 \mathrm{~min}$ at $12,000 \mathrm{~g}$ and the supernatant was collected. The protein concentrations were measured using the Pierce BCA Protein Assay kit (Thermo Fisher Scientific, Waltham, MA, USA). Then, an equal volume of supernatant was incubated with thiobarbituric acid buffer at $100^{\circ} \mathrm{C}$ for $15 \mathrm{~min}$. Subsequently, the absorbance was measured at $532 \mathrm{~nm}$ using a VERS Amax Microplate Reader (Molecular Devices Corp, Sunnyvale, CA, USA). The values were expressed as MDA (nmol/mg protein). All assays were conducted in triplicate.

\section{RNA Interference}

A small interfering RNA (siRNA) targeting human p38 $\alpha$ (GCCCAUAAGGCCAGAAACUtt) and a negative control siRNA (siNC, UUCUCCGAACGUGUCACGUtt) were obtained from GenePharma (Shanghai, China). Cells were transfected with the siRNAs using Lipofectamine RNAiMAX (\#13778150, Invitrogen) for $48 \mathrm{~h}$ in accordance with the manufacturer's instructions. The knockdown efficiencies of the siRNAs were determined by Western blotting analysis.

\section{Statistical Analysis}

Comparisons between two groups were made through unpaired Student's $t$-test. Comparisons of parameters among multiple groups were made using one-way ANOVA followed by Tukey's test. The data are shown as the mean \pm standard deviation (S.D.). Each experiment was repeated at least three times. All statistical tests were conducted using Prism 6 software (GraphPad Software, San Diego, CA, USA). ${ }^{* *} p<0.05$ indicates differences.

\section{Results}

\section{Physical Properties and Cellular Uptake of CuONPs}

The energy dispersive spectroscopy and dynamic light scattering properties of CuONPs were examined in our previous study. ${ }^{20}$ The morphology of CuONPs in deionized water was
A

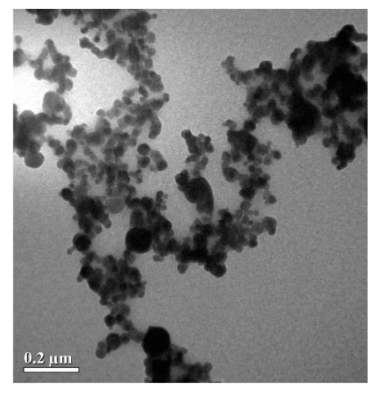

B

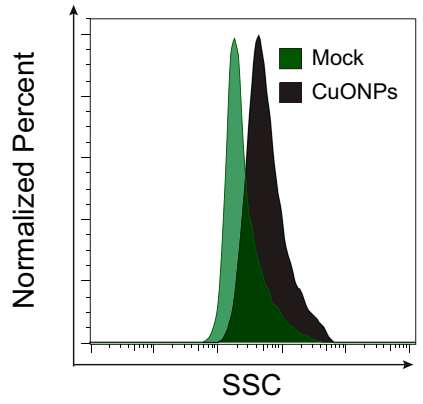

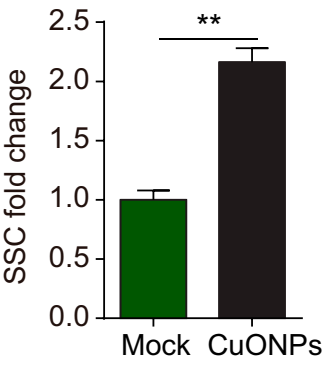

C
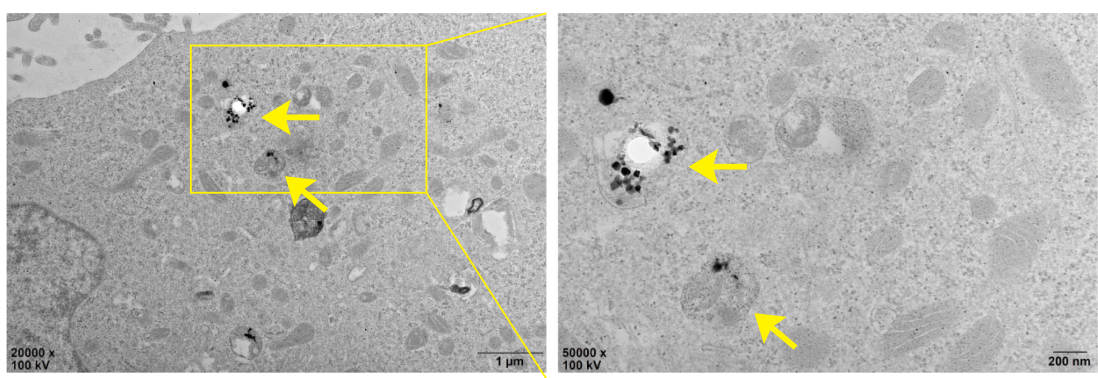

Figure I Physical properties and cellular uptake of CuONPs. (A) A representative transmission electron microscope (TEM) image of CuONPs dissolved in distilled water. (B) Representative FACS data for SSC intensity of HUVECs treated with $20 \mu \mathrm{g} / \mathrm{mL}$ CuONPs for $12 \mathrm{~h}$. Mock, untreated HUVECs. Unpaired Student's $t$-tests were performed for statistical analysis. ${ }^{* *} p<0.05$ versus untreated HUVECs. (C) Representative TEM images of HUVECs treated with $20 \mu \mathrm{g} / \mathrm{mL}$ CuONPs for $12 \mathrm{~h}$. Yellow arrows indicate lysosomal deposition of CuONPs. 
characterized by transmission electron microscope (TEM). TEM images showed that CuONPs was subglobose shape with an average size of $<50 \mathrm{~nm}$ (Figure 1A). Cellular uptake of CuONPs was confirmed by FACS assay as described previously. ${ }^{28}$ FACS results showed that CuONPs induced an increase of SSC intensity of HUVECs, indicating the endocytosis of CuONPs into HUVECs (Figure 1B). Furthermore, TEM images showed that CuONPs mainly aggregated in the lysosomes of HUVECs (Figure 1C).

\section{CuONPs Triggered DNA Damage and Cell Death in HUVECs}

Cytotoxic effects of CuONPs on vascular endothelial cells were investigated with MTS assay. The results showed that CuONPs reduced HUVECs viability in a dose-dependent manner (Figure 2A). FACS assay after Calcein AM labeling also confirmed that $\mathrm{CuONPs}$ exposure caused more than $50 \%$ of cell death in HUVECs (Figure 2B). Comet assay results revealed that CuONPs treatment caused DNA damage in HUVECs (Figure 2C). DNA damage in CuONPs-treated
HUVECs were further evaluated by $\gamma \mathrm{H} 2 \mathrm{AX}$ foci formation (a sensitive molecular marker of DNA damage). The results from immunofluorescence staining demonstrated that $\mathrm{CuONPs}$ treatment resulted in the formation of $\gamma \mathrm{H} 2 \mathrm{AX}$ foci in the nuclei of HUVECs (Figure 2D). Furthermore, we showed that the phosphorylation levels of ATR, ATM, p53 and H2AX increased obviously, suggesting that CuONPs caused DNA damage in HUVECs in an ATM/ATR-p53dependent manner (Figure 2E and Figure S1A-D). Collectively, these results indicate that CuONPs exposure trigger DNA damage and cell death in HUVECs.

\section{CuONPs Induced Oxidative Stress in HUVECs}

To investigate whether CuONPs trigger oxidative stress in HUVECs, the level of superoxide anions in CuONPs-treated HUVECs were detected using the fluorescent probe DHE and MitoSOX. FACS data showed that CuONPs exposure significantly increased intracellular level of superoxide anions in HUVECs (Figure $3 \mathrm{~A}$ and $\mathrm{B}$ and Figure S2A-B).

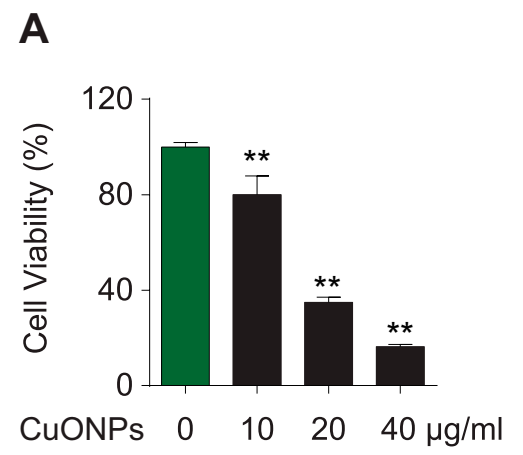

C
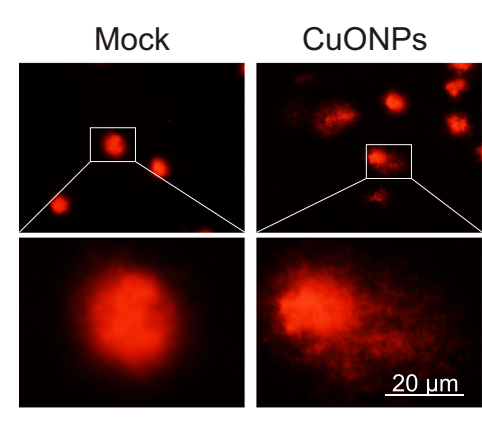

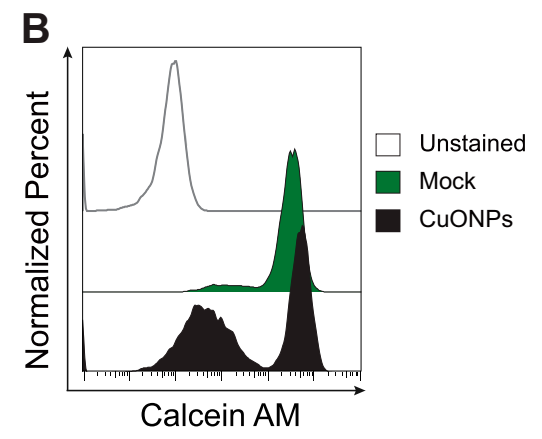

D

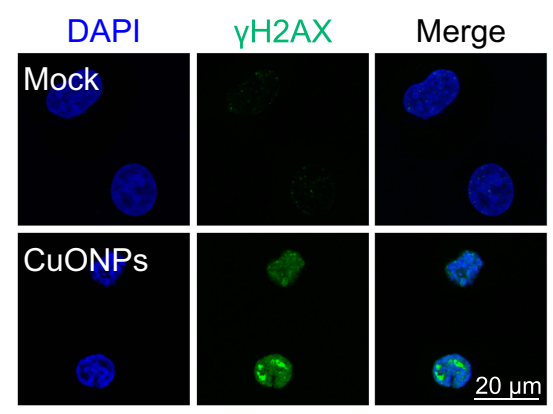

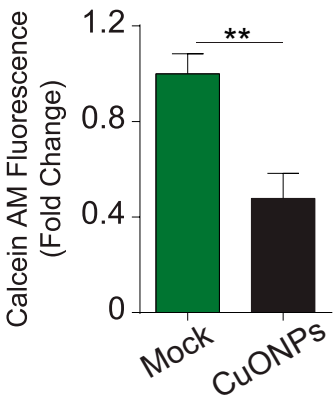

E

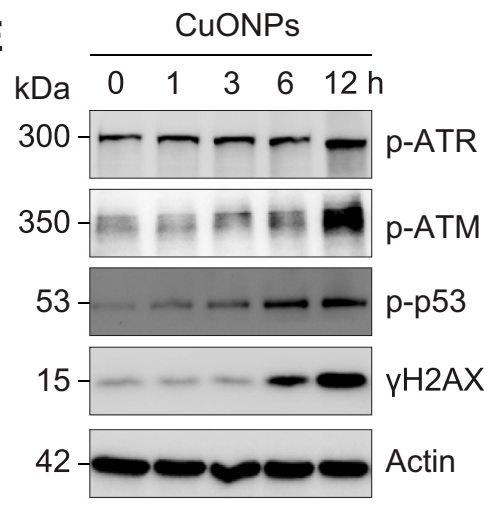

Figure 2 DNA damage and cell death in CuONPs-treated HUVECs. (A) MTS assay of HUVECs treated with different concentrations of CuONPs (0, I0, 20, or $40 \mu \mathrm{g} / \mathrm{mL})$ for 24 h. (B) Representative FACS data for CuONPs-treated HUVECs after Calcein AM (live cell fluorescent probe) staining. (C) Comet assay to evaluate CuONPs-induced DNA damage in HUVECs. (D) Immunofluorescence assay of $\gamma \mathrm{H} 2 \mathrm{AX}$ foci formation in CuONPs-treated HUVECs. (E) Western blotting assay of phospho-ATR, phosphoATM, phospho-p53 and phospho-H2AX $(\gamma \mathrm{H} 2 \mathrm{AX})$ in HUVECs treated with CuONPs $(20 \mu \mathrm{g} / \mathrm{mL})$ for 0, I, 3, 6 and I 2 h. Actin was used as a loading control. In $($ A $)$, one-way ANOVA followed by Tukey's test was performed for statistical analysis. In (B), unpaired Student's $t$-tests were performed for statistical analysis. $* * p<0.05$ versus untreated HUVECs. 
A

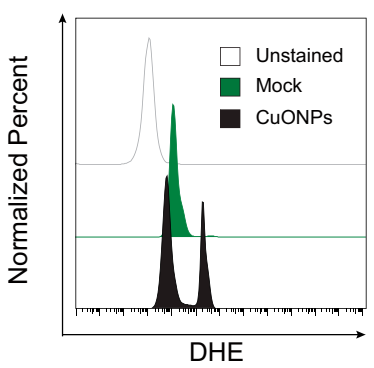

D

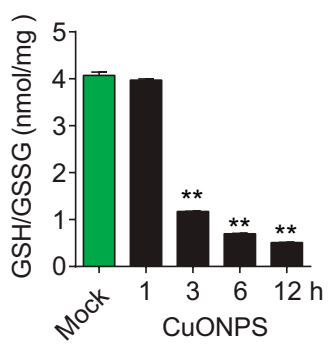

B

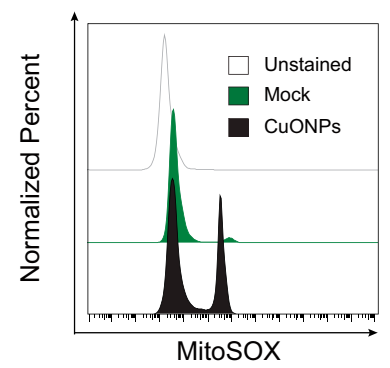

E

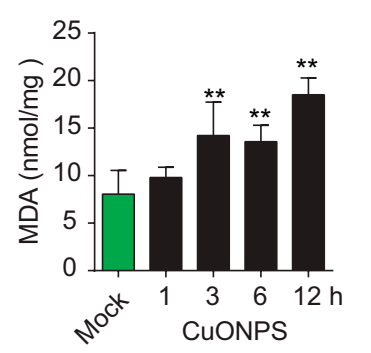

C

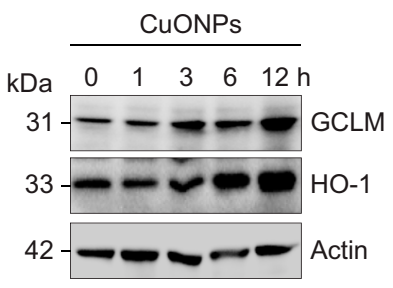

Figure 3 CuONPs treatment caused oxidative stress in HUVECs. (A) Representative FACS data for cellular superoxide anions in CuONPs-treated HUVECs after staining with the fluorescent probe DHE. (B) Representative FACS data for mitochondrial superoxide anions in CuONPs-treated HUVECs after staining with the fluorescent probe MitoSOX. (C) Western blotting analysis of protein levels of GCLM and HO-I. Actin was used as a loading control. (D) GSH/GSSG ratio assays of HUVECs treated with CuONPs $(20 \mu \mathrm{g} / \mathrm{mL})$ for $0, \mathrm{I}, 3,6$ and I $2 \mathrm{~h}$. (E) Lipid peroxidation (MDA) assay of HUVECs treated with CuONPs (20 $\mu \mathrm{g} / \mathrm{mL})$ for 0, I, 3, 6 and I2 h. In (D) and (E), one-way ANOVA followed by Tukey's test was performed for statistical analysis. $*_{p} p<0.05$ versus untreated HUVECs.

Then, the protein levels of antioxidant enzymes GCLM and HO-1 in HUVECs were determined. Our results revealed that GCLM and HO-1 were upregulated in CuONPs-treated HUVECs in a dose-dependent manner (Figure 3C and Figure S2C-D). Analysis of GSH and GSSG showed that CuONPs caused a decrease in GSH/GSSG ratio in HUVECs (Figure 3D). As lipid oxidation occurs when cells undergo oxidative stress, the cellular level of lipid oxidation in CuONPs-treated HUVECs were examined. The results showed that CuONPs also caused MDA accumulation in HUVECs (Figure 3E). In brief, our results suggest that CuONPs induced oxidative stress in HUVECs.

\section{CuONPs-Induced Oxidative Stress Mediated DNA Damage and Cell Death in HUVECs}

To determine the role of oxidative stress in CuONPstreated HUVECs, we pretreated HUVECs with antioxidant N-Acetyl-L-cysteine (NAC). The results demonstrated that NAC ameliorated CuONPs-induced cell death in a dosedependent manner (Figure 4A). Calcein AM assay also showed that NAC rescued HUVECs from CuONPsinduced cell death (Figures 4B and $\underline{\mathrm{S} 3 \mathrm{~A}}$ ). Moreover, NAC significantly prevented the CuONPs-induced upregulation of phosphorylation levels of ATR, ATR, p53 and $\mathrm{H} 2 \mathrm{AX}$ in HUVECs (Figures $4 \mathrm{C}$ and S3B-E). NAC pretreatment also reduced $\gamma \mathrm{H} 2 \mathrm{AX}$ foci formation in HUVECs induced by CuONPs (Figure 4D). All these data indicate that oxidative stress-mediated DNA damage and cell death in CuONPs-treated HUVECs.

\section{p38 MAPK Contributed to CuONPs-Induced DNA Damage and Cell Death in HUVECs}

The p38 MAPK is activated in response to cellular stress. In this study, we showed that CuONPs also induced a robust activation of p38 MAPK and subsequent phosphorylation of HSP27 and ATF-2, two downstream effectors of the p38 MAPK pathway (Figures 5A and $\underline{\mathrm{S} 4 \mathrm{~A}}$ ). Next, we examined whether p38 MAPK plays a role in CuONPs toxicity on vascular endothelial cells. The results showed that $\mathrm{p} 38 \alpha$ MAPK knockdown by siRNA prevented CuONPs-induced changes of the phosphorylation levels ATR, H2AX, HSP27 and ATF-2 in HUVECs (Figures $5 \mathrm{~B}$ and $\mathrm{C}$ and $\mathrm{S} 4 \mathrm{~B}-\mathrm{C}$ ). Then, we demonstrated that p38 $\alpha$ MAPK knockdown significantly rescued HUVECs from CuONPs-induced cell death in a dose-dependent manner (Figure 5D). Collectively, these data demonstrate that p38 MAPK 
A

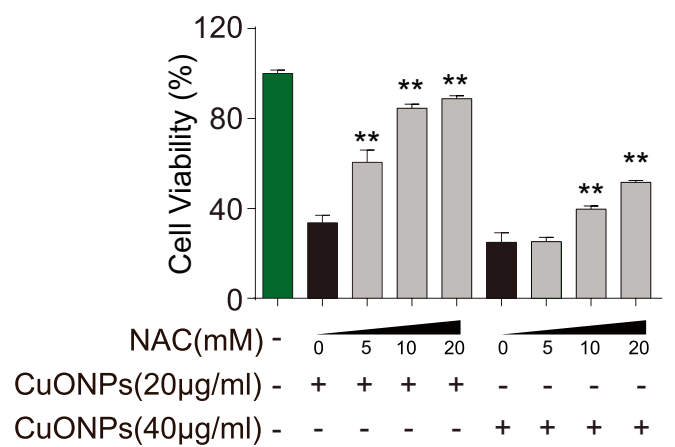

B

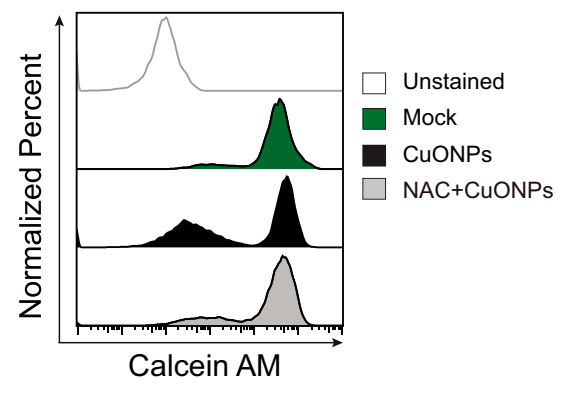

C

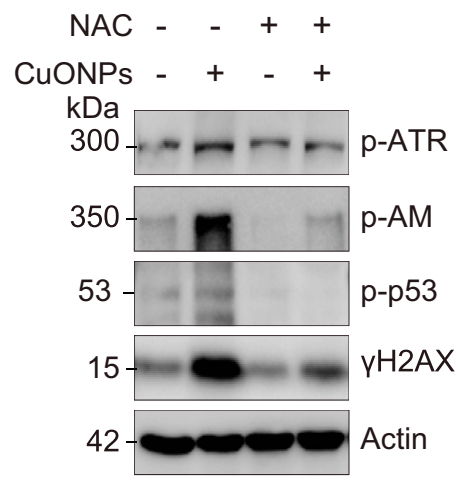

D

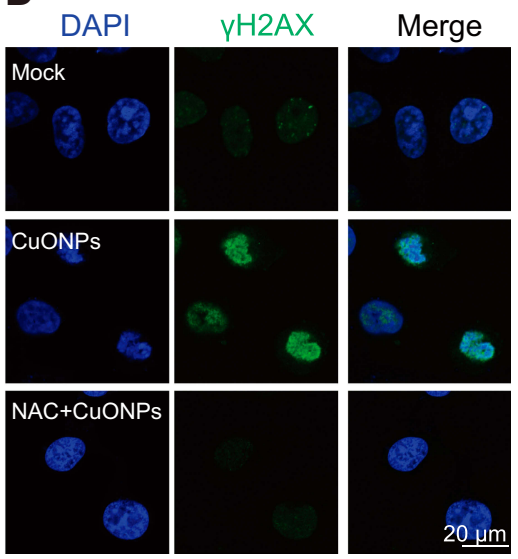

Figure 4 Oxidative stress-mediated DNA damage and cell death in CuONPs-treated HUVECs. (A) MTS assay of HUVEC cell viability after pretreatment with different concentrations of NAC $(0,5,10$ or $20 \mathrm{mM})$ for I h and then treatment with CuONPs (20 or $40 \mu \mathrm{g} / \mathrm{mL})$ for 24 h. one-way ANOVA followed by Tukey's test was performed for statistical analysis. ${ }^{* *} p<0.05$. (B) Representative FACS data for Calcein AM fluorescence of CuONPs-treated HUVECs. (C) Western blotting assay of phospho-ATR, phospho-ATM, phospho-p53 and $\gamma \mathrm{H} 2 \mathrm{AX}$ in HUVECs, which were pretreated with or without NAC ( $10 \mathrm{mM})$ for I h and then treated with CuONPs $(20 \mu \mathrm{g} / \mathrm{mL})$ for 12 h. Actin was used as a loading control. (D) Immunofluorescence assay of $\gamma \mathrm{H} 2 \mathrm{AX}$ foci formation in CuONPs-treated HUVECs.

signaling is directly involved in CuONPs-induced DNA damage and cell death in HUVECs.

\section{The Copper lons Chelator Tetrathiomolybdate Alleviated P38 MAPK-Mediated DNA Damage and Cell Death in CuONPs-Treated HUVECs}

In our previous study, we demonstrated that CuONPs exposure resulted in the release of copper ions in HUVECs. ${ }^{20}$ Therefore, in the current study, we determined whether the released copper ions mediate p38 MAPK-mediated DNA damage and cell death in CuONPs-treated HUVECs. We showed that copper ions chelator TTM prevent the upregulation of superoxide anions in CuONPs-treated HUVECs (Figures 6A and B and S5A-B). The results from MDA assay showed that TTM alleviated CuONPs-induced oxidative stress in HUVECs (Figure 6C). In addition, TTM inhibited the activation of $\mathrm{p} 38 \mathrm{MAPK}$, indicated by the reduction of p38 MAPK, HSP27 and ATF-2 phosphorylation in TTMtreated HUVECs (Figures 6D and S5C). Furthermore, the chelation of copper ions also attenuated CuONPs-induced $\gamma \mathrm{H} 2 \mathrm{AX}$ foci formation and the upregulation of ATR, ATM, p53 and H2AX phosphorylation in HUVECs (Figures 6E and $\mathrm{F}$ and $\mathrm{S} 5 \mathrm{D})$. Finally, we showed that the chelation of copper ions rescued HUVECs from CuONPs-induced cell death in a dose-dependent manner (Figure 6G). Calcein AM assay further confirmed that TTM treatment reduced HUVECs death induced by CuONPs (Figures $6 \mathrm{H}$ and $\underline{\mathrm{S} 5 \mathrm{E}}$ ). Collectively, these findings indicate that copper ionssuperoxide anions-p38 MAPK-DNA damage axis mediated CuONPs-induced cell death in HUVECs.

\section{Discussion}

CuONPs have been widely applied in multiply areas. ${ }^{29,30}$ Air-blood barrier translocation of inhaled CuONPs may directly modulate endothelial dysfunction and the pathogenesis of atherosclerosis, ${ }^{17}$ thus it is important to elucidate 
A

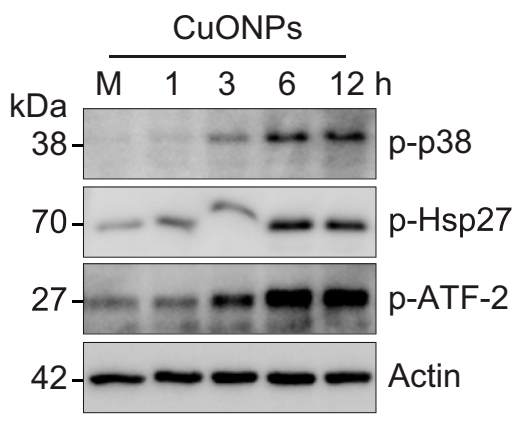

C

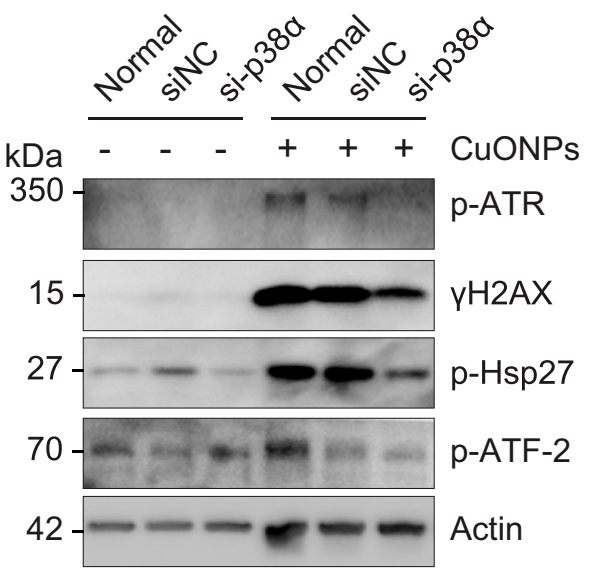

B

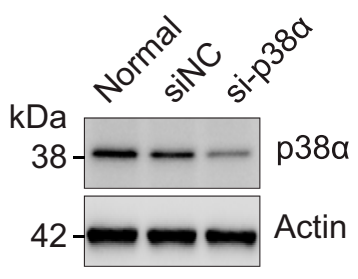

D

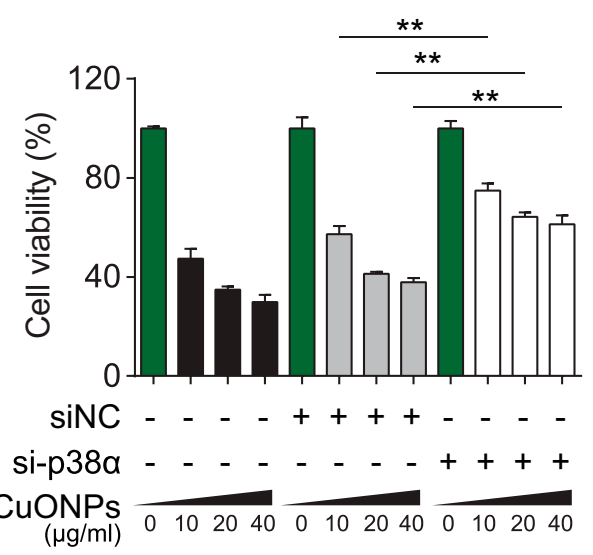

Figure 5 p38 MAPK was involved in DNA damage and cell death in CuONPs-treated HUVECs. (A) Western blotting assay of phospho-p38, phospho-Hsp27 and phosphorATF-2 in HUVECs treated with CuONPs $(20 \mu \mathrm{g} / \mathrm{mL})$ for $0,1,3,6$ and I2 h. Actin was used as a loading control. (B) Western blotting assay of $\mathrm{p} 38 \alpha$ in HUVECs transfected with $75 \mathrm{nM}$ siNC (negative control siRNA) or si-p38 $\alpha$ for $48 \mathrm{~h}$. Actin was used as a loading control. (C) Western blotting assay of phospho-ATR, $\gamma \mathrm{H} 2 \mathrm{AX}$, phospho-Hsp27 and phospho-ATF-2 in HUVECs, which were transfected with siNC or si-p38 $\alpha$ for $48 \mathrm{~h}$ and then treated with CuONPs (20 $\mu \mathrm{g} / \mathrm{mL})$ for $12 \mathrm{~h}$. Actin was used as a loading control. (D) MTS assay of HUVEC after transfection with siNC or si-p38 $\alpha$ for $48 \mathrm{~h}$ and then treatment with different concentrations of $\mathrm{CuONPs}(0,10,20 \mathrm{or} 40 \mu \mathrm{g} / \mathrm{mL})$ for $24 \mathrm{~h}$. One-way ANOVA followed by Tukey's test was performed for statistical analysis. $* * p<0.05$ versus untreated HUVECs.

the molecular mechanism underlying CuONPs-induced vascular endothelial dysfunction.

In our previous studies, we showed that CuONPs exposure induced excessive accumulation of superoxide anions in vascular endothelial cells, which are mainly attributed to CuONPs lysosomal deposition-induced lysosomal dysfunction and disruption of protective mitophagy. ${ }^{20,25}$ In the current study, we further confirmed that CuONPs induced oxidative stress in HUVECs, indicated by the increase of cellular levels of superoxide anions, the upregulation of protein levels of HO-1 and GCLM, the elevation of the levels of MDA, but the reduction of GSH/GSSG ratio (Figure 3). Furthermore, we revealed that CuONPsinduced oxidative stress directly triggered DNA damage and cell death in HUVECs (Figures 2 and 4). This finding is consistent with previous studies reporting that oxidative stress is a mechanism of CuONPs toxicity. ${ }^{31-33}$ Oxidative stress may lead to irreversible oxidation of proteins, lipids and nucleic acids, consequently trigger DNA damage and cell death in HUVECs.

Copper is one of the important metal elements in maintaining homeostasis. ${ }^{34}$ Copper deficiency can result in a wide variety of clinical manifestations including impaired growth, neurological dysfunction and other possible abnormalities. ${ }^{35}$ Conversely, copper overload can also cause many diseases such as Wilson's disease ${ }^{36}$ and Alzheimer's disease. ${ }^{37}$ Previous studies have demonstrated that the toxicity of metal nanomaterials may originate from the nanoparticles themselves, the released metal ions or a combination of both. ${ }^{18,38}$ In our previous study, we showed that the copper ions released from CuONPs was involved in CuONPs-induced cell death. ${ }^{20}$ In the current study, we confirmed that TTM, a copper chelator for the treatment of Wilson's disease, ${ }^{39}$ alleviated CuONPs-induced lipid oxidation, HUVECs DNA damage 
A

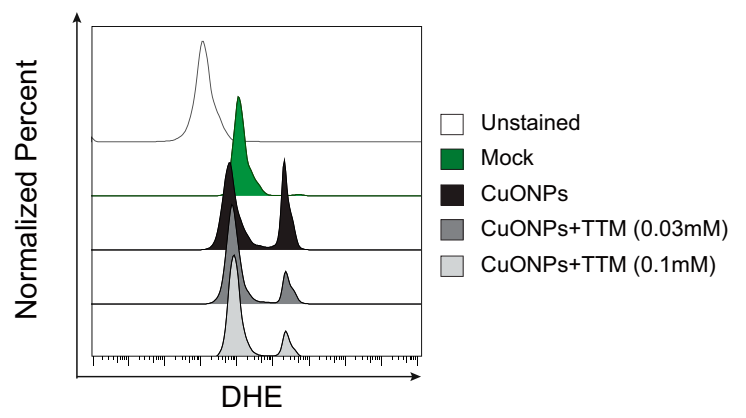

B

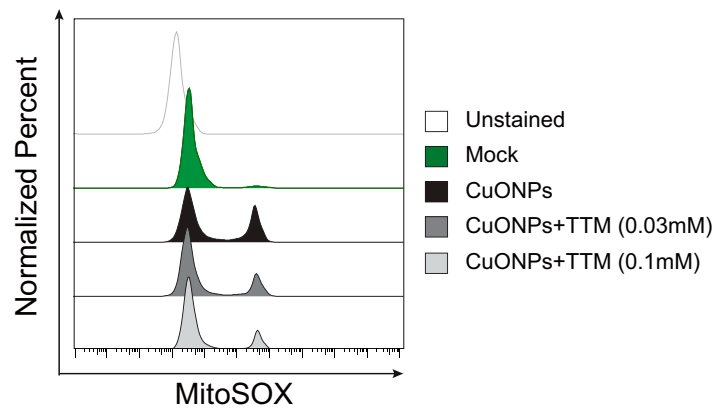

E

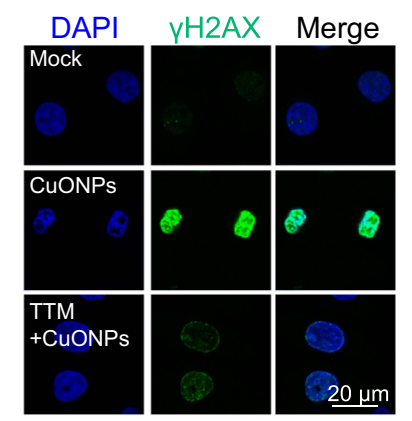

C

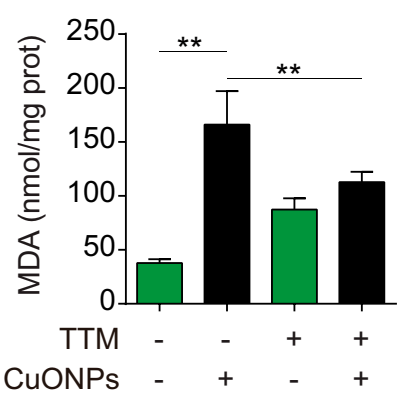

F

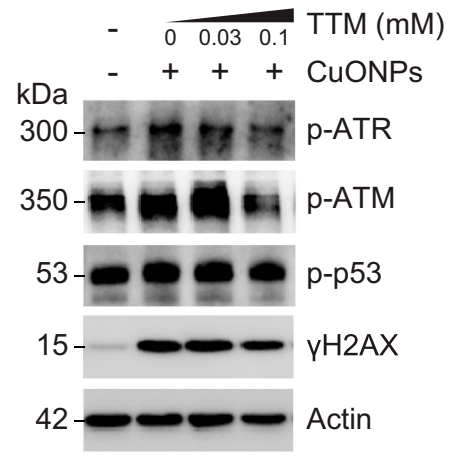

D

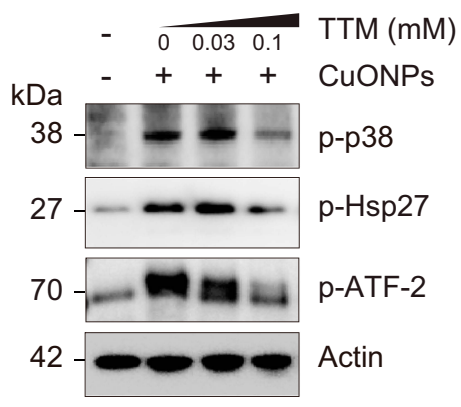

G

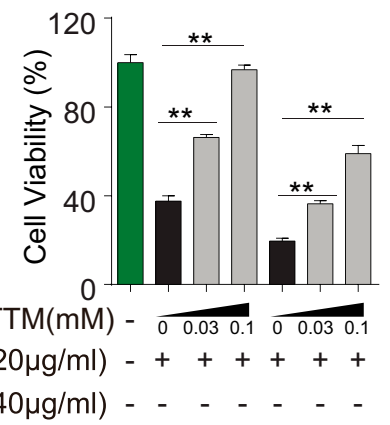

H

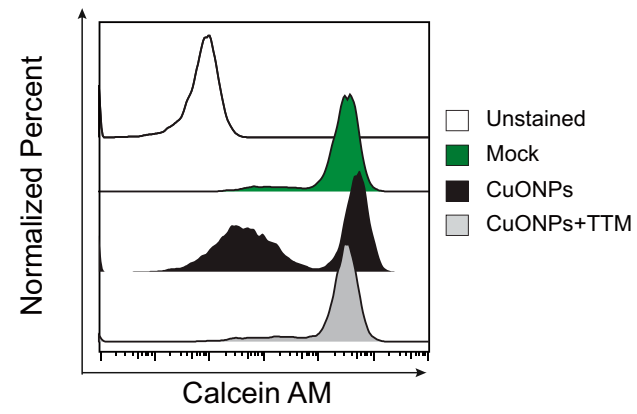

Figure 6 Copper ions chelator TTM alleviated HUVECs DNA damage and cell death induced by CuONPs. (A) and (B) Representative FACS data regarding cellular superoxide anions and mitochondrial superoxide anions after staining with the fluorescent probe DHE and MitoSOX, respectively. HUVECs were pretreated with different concentrations of the copper ions chelator TTM $(0,0.03$ and $0.1 \mathrm{mM})$ for I h and then treated with CuONPs $(20 \mu \mathrm{g} / \mathrm{mL})$ for $12 \mathrm{~h}$. (C) Lipid peroxidation (MDA) assay of HUVECs pretreated with or without TTM $(0.1 \mathrm{mM})$ for I h and then treated with CuONPs $(20 \mu \mathrm{g} / \mathrm{mL})$ for $12 \mathrm{~h}$. (D) Western blotting assay of phosphor-p38, phosphorHsp27 and phosphor-ATF-2 in HUVECs, pretreated with or without TTM $(0,0.03$, and $0.1 \mathrm{mM})$ for $1 \mathrm{~h}$ and then treated with CuONPs $(20 \mu \mathrm{g} / \mathrm{mL})$ for $12 \mathrm{~h}$. Actin was used as a loading control. (E) Immunofluorescence assay of the level of $\gamma \mathrm{H} 2 \mathrm{AX}$ in CuONPs-treated HUVECs, pretreated with or without TTM (0.I mM). (F) Western blotting assay of phospho-ATR, phospho-ATM, phospho-p53 and $\gamma \mathrm{H} 2 \mathrm{AX}$ in HUVECs, which were pretreated with or without TTM (0, 0.03 , and 0.1 mM) for I h and then treated with CuONPs $(20 \mu \mathrm{g} / \mathrm{mL})$ for $12 \mathrm{~h}$. Actin was used as a loading control. (G) MTS assay of HUVECs viability after pretreatment with different concentrations of the copper ions chelator TTM $(0,0.03$, and $0.1 \mathrm{mM})$ for I h and then treatment with CuONPs $(20$ or $40 \mu \mathrm{g} / \mathrm{mL})$ for $24 \mathrm{~h}$. (H) Representative FACS data for Calcein AM fluorescence in HUVECs, which were pretreated with or without TTM $(0.1 \mathrm{mM})$ for I h and then treated with CuONPs $(20 \mu \mathrm{g} / \mathrm{mL})$ for $12 \mathrm{~h}$. In $(\mathbf{C})$ and $(\mathbf{G})$, one-way ANOVA followed by Tukey's test was performed for statistical analysis. $* * p<0.05$.

and cell death in HUVECs (Figure 6E-H). Our data suggest that the release of copper ions were the upstream factor of CuONPs-induced DNA damage and cell death in HUVEC, indicating that TTM may be an effective treatment for CuONPs-cardiovascular diseases.
The exact roles of copper ions in CuONPs toxicity are also explored in the present study. We demonstrated that the release of copper ions was involved in oxidative stress response in CuONPs-treated HUVECs (Figure 6A-C). It has been reported that the levels of serum copper ions are increased 


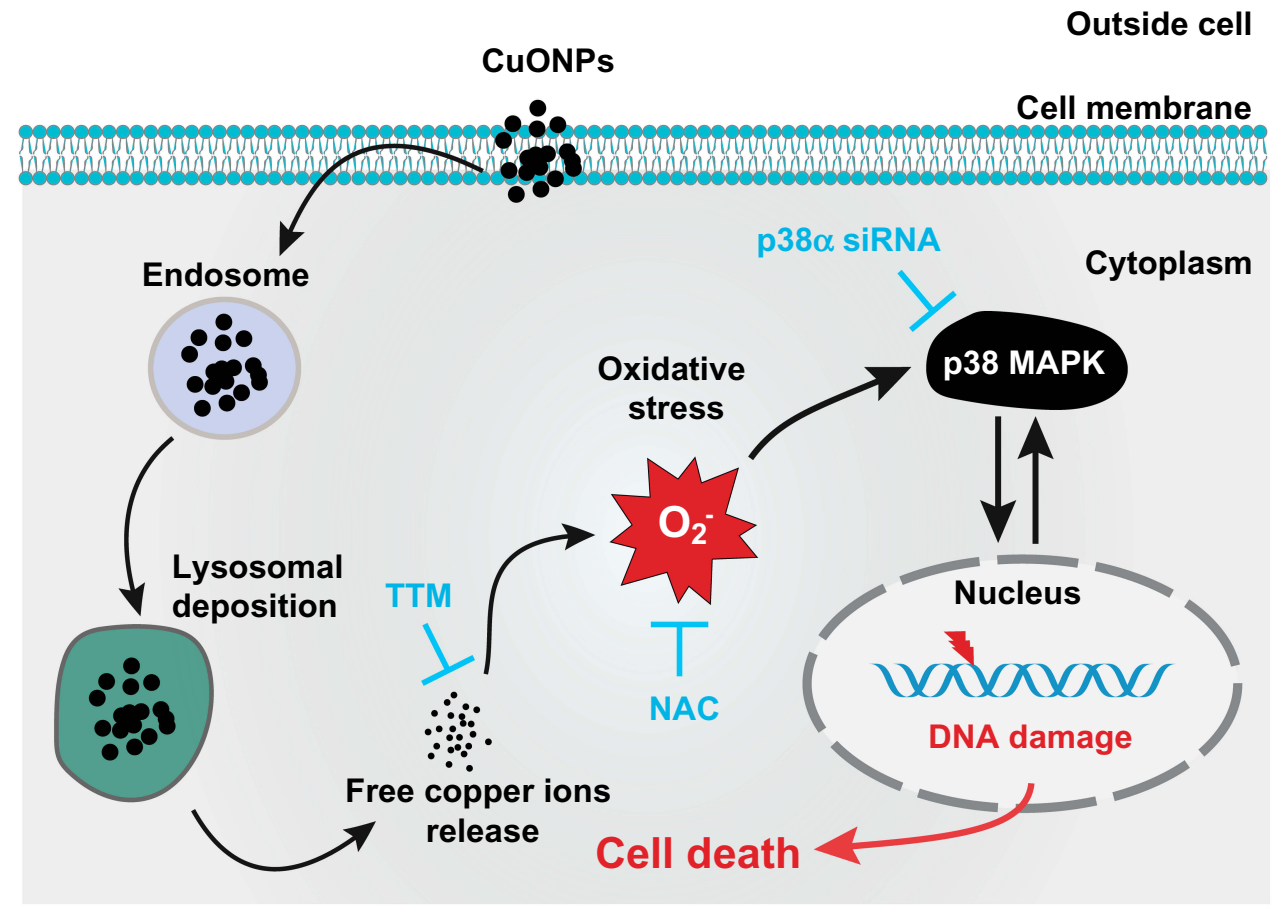

HUVEC Vascular endothelial cell

Figure 7 Schematic representation of the role of released copper ions in P38 MAPK-mediated DNA damage and cell death in CuONPs-treated HUVECs. Cellular uptake and lysosomal deposition of CuONPs lead to the release of copper ions from CuONPs, which triggers oxidative stress response and $\mathrm{p} 38$ MAPK signaling activation. The activation of p38 MAPK signaling pathway contributes to CuONPs-induced DNA damage and cell death in HUVECs. Meanwhile, CuONPs-induced DNA damage may feedback-regulate $\mathrm{P} 38$ MAPK signaling pathway, resulting in aggravation of DNA damage and cell death in HUVECs.

under inflammatory conditions to trigger oxidative stress responses that activate inflammatory responses. ${ }^{40}$ Conversely, copper ions selective chelators can alleviate copper-induced oxidative stress. ${ }^{41}$ The p38 MAPK signaling pathway has been implicated in cellular responses including inflammation, metabolism, cell death, development, cell differentiation, senescence, and tumorigenesis. ${ }^{42,43}$ The p38 MAPK signaling pathway has been implicated in cellular responses to exposure to CuONPs in vivo and in vitro model. $^{25,44,45}$ In this study, we further determined that p38 MAPK pathway activation was implicated in copper ionsinduced oxidative stress in HUVECs (Figure 6D). All these data indicate that the CuONPs exposure causes a significant increase of cellular copper ions, which induce oxidative stress and activate MAPK pathways. Nevertheless, the upstream regulators and downstream effectors of p38 MAPK in CuONPs-treated HUVECs need further investigations.

It is well documented that p38 MAPK is activated in response to DNA double strand breaks (DSBs). ${ }^{46} \mathrm{DSBs}$, in turn, promoted nuclear translocation of p38 MAPK to facilitate the phosphorylation of $\mathrm{p} 38$ MAPK downstream effectors to induce $\mathrm{G} 2 / \mathrm{M}$ cell cycle checkpoint and DNA repair. ${ }^{47}$
There is increasing evidence that p38 MAPK signaling pathway is also involved in nanoparticle-induced cytotoxicity. Gold nanoparticles exposure induced mechanical stress and activation of p38 MAPK pathway in mesenchymal stem cells. $^{48}$ In the current study, our results revealed that CuONPs increased the phosphorylation levels of p38 MAPK and its downstream targets Hsp27 and ATF-2, while p38 MAPK knockdown could prevent CuONPs-induced cytotoxicity in HUVECs (Figure 5). This supports the previous findings showing that silver nanoparticles exposure activates p38 MAPK through the nuclear factor-E2-related factor-2 and nuclear factor-kappaB signaling pathways, subsequently inducing DNA damage, cell cycle arrest and apoptosis. These findings also indicate that p38 MAPK may play dual roles in nanoparticle-induced cytotoxicity and genotoxicity. Nanoparticle-induced DNA damage stress may activate p38 MAPK to induce DNA repair or trigger cell death directly.

In summary, our findings demonstrate that the release of copper ions contributes to CuONPs-induced vascular endothelial cell death. The released copper ions induced oxidative stress and the activation of the p38 MAPK signaling pathway in HUVECs, while the chelation of copper ions significantly 
reduced the oxidative stress response and inhibited the activation of p38 MAPK signaling. Furthermore, CuONPs-induced oxidative stress may trigger DNA damage and apoptotic cell death in HUVECs through the p38 MAPK signaling pathway, because p38 $\alpha$ knockdown significantly rescued CuONPsinduced oxidative DNA damage and HUVECs death (Figure 7). These findings provide an insight into the underlying links between the release of copper ions, oxidative stress and activation of downstream p38 MAPK signaling in CuONPs-induced cytotoxicity in the cardiovascular system.

\section{Acknowledgments}

This work was supported by the National Natural Science Foundation of China (81500343 and 81903358), the Chongqing Research Program of Basic Research and Frontier Technology (cstc2018jcyjAX0355, cstc2017jcyjA X0162) and the Science and Technology Project Affiliated to the Education Department of Chongqing, China (KJQN 201800434 and KJQN201900421).

\section{Disclosure}

The authors report no conflicts of interest in this work.

\section{References}

1. Jiang XC, Herricks T, Xia YN. CuO nanowires can be synthesized by heating copper substrates in air. Nano Lett. 2002;2(12):1333-1338. doi: $10.1021 / \mathrm{n} 10257519$

2. Diao FY, Tian FH, Liang WS, Feng HL, Wang YQ. Mechanistical investigation on the self-enhanced photocatalytic activity of $\mathrm{CuO} /$ $\mathrm{Cu} 2 \mathrm{O}$ hybrid nanostructures by density functional theory calculations. Phys Chem Chem Phys. 2016;18(40):27967-27975. doi:10.1039/C6CP03977D

3. Choi KJ, Jang HW. One-dimensional oxide nanostructures as gas-sensing materials: review and issues. Sensors. 2010;10 (4):4083-4099. doi:10.3390/s100404083

4. Oberdorster G. Pulmonary effects of inhaled ultrafine particles. Int Arch Occ Env Hea. 2001;74(1):1-8. doi:10.1007/s004200000185

5. Xu M, Fujita D, Kajiwara S, et al. Contribution of physicochemical characteristics of nano-oxides to cytotoxicity. Biomaterials. 2010;31 (31):8022-8031. doi:10.1016/j.biomaterials.2010.06.022

6. Karlsson HL, Cronholm P, Gustafsson J, Moller L. Copper oxide nanoparticles are highly toxic: a comparison between metal oxide nanoparticles and carbon nanotubes. Chem Res Toxicol. 2008;21 (9):1726-1732. doi:10.1021/tx800064j

7. Sun T, Yan Y, Zhao Y, Guo F, Jiang C, Srinivasula SM. Copper oxide nanoparticles induce autophagic cell death in A549 cells. PLoS One. 2012;7(8):e43442. doi:10.1371/journal.pone.0043442

8. Wongrakpanich A, Mudunkotuwa IA, Geary SM, et al. Size-dependent cytotoxicity of copper oxide nanoparticles in lung epithelial cells. Environ Sci Nano. 2016;3(2):365-374. doi:10.1039/C5EN00271K

9. Hanagata N, Zhuang F, Connolly S, Li J, Ogawa N, Xu M. Molecular responses of human lung epithelial cells to the toxicity of copper oxide nanoparticles inferred from whole genome expression analysis. ACS Nano. 2011;5(12):9326-9338. doi:10.1021/nn202966t
10. Laha D, Pramanik A, Maity J, et al. Interplay between autophagy and apoptosis mediated by copper oxide nanoparticles in human breast cancer cells MCF7. Biochim Biophys Acta. 2014;1840(1):1-9. doi:10.1016/j.bbagen.2013.08.011

11. Rodhe Y, Skoglund S, Odnevall Wallinder I, Potacova Z, Moller L. Copper-based nanoparticles induce high toxicity in leukemic HL60 cells. Toxicol In Vitro. 2015;29(7):1711-1719. doi:10.1016/j. tiv.2015.05.020

12. Piret JP, Jacques D, Audinot JN, et al. Copper(II) oxide nanoparticles penetrate into HepG2 cells, exert cytotoxicity via oxidative stress and induce pro-inflammatory response. Nanoscale. 2012;4 (22):7168-7184

13. Thit A, Selck H, Bjerregaard HF. Toxic mechanisms of copper oxide nanoparticles in epithelial kidney cells. Toxicol in Vitro. 2015;29 (5):1053-1059. doi:10.1016/j.tiv.2015.03.020

14. Bulcke F, Thiel K, Dringen R. Uptake and toxicity of copper oxide nanoparticles in cultured primary brain astrocytes. Nanotoxicology. 2014;8(7):775-785. doi:10.3109/17435390.2013.829591

15. Franklin BA, Brook R, Pope III CA. Air pollution and cardiovascular disease. Curr Probl Cardiol. 2015;40(5):207-238. doi:10.1016/j. cpcardiol.2015.01.003

16. Coultas L, Chawengsaksophak K, Rossant J. Endothelial cells and VEGF in vascular development. Nature. 2005;438(7070):937. doi:10.1038/nature04479

17. Miller MR, Raftis JB, Langrish JP, et al. Inhaled nanoparticles accumulate at sites of vascular disease. ACS Nano. 2017;11 (5):4542-4552. doi:10.1021/acsnano.6b08551

18. Jiang X, Tang Q, Zhang J, et al. Autophagy-dependent release of zinc ions is critical for acute lung injury triggered by zinc oxide nanoparticles. Nanotoxicology. 2018;12(9):1068-1091. doi:10.1080/ 17435390.2018 .1513094

19. Zhang J, Qin X, Wang B, et al. Zinc oxide nanoparticles harness autophagy to induce cell death in lung epithelial cells. Cell Death Dis. 2017;8(7):e2954. doi:10.1038/cddis.2017.337

20. Zhang J, Zou Z, Wang B, et al. Lysosomal deposition of copper oxide nanoparticles triggers HUVEC cells death. Biomaterials. 2018;161:228-239. doi:10.1016/j.biomaterials.2018.01.048

21. von Moos N, Slaveykova VI. Oxidative stress induced by inorganic nanoparticles in bacteria and aquatic microalgae-state of the art and knowledge gaps. Nanotoxicology. 2014;8(6):605-630. doi:10.3109/ 17435390.2013.809810

22. Schieber M, Chandel NS. ROS function in redox signaling and oxidative stress. Curr Biol. 2014;24(10):R453-R462. doi:10.1016/j. cub.2014.03.034

23. Wan R, Mo Y, Feng L, Chien S, Tollerud DJ, Zhang Q. DNA damage caused by metal nanoparticles: involvement of oxidative stress and activation of ATM. Chem Res Toxicol. 2012;25(7):1402-1411. doi:10.1021/tx200513t

24. Sharma V, Anderson D, Dhawan A. Zinc oxide nanoparticles induce oxidative DNA damage and ROS-triggered mitochondria mediated apoptosis in human liver cells (HepG2). Apoptosis. 2012;17 (8):852-870. doi:10.1007/s10495-012-0705-6

25. Zhang Y, Ding Z, Zhao G, et al. Transcriptional responses and mechanisms of copper nanoparticle toxicology on zebrafish embryos. J Hazard Mater. 2018;344:1057-1068. doi:10.1016/j. jhazmat.2017.11.039

26. Qin X, Zhang J, Wang B, Xu G, Zou Z. LAMP-2 mediates oxidative stress-dependent cell death in Zn2+-treated lung epithelium cells. Biochem Biophys Res Commun. 2017;488(1):177-181. doi:10.1016/ j.bbrc.2017.05.030

27. Chen C, Jiang X, Lai Y, Liu Y, Zhang Z. Resveratrol protects against arsenic trioxide-induced oxidative damage through maintenance of glutathione homeostasis and inhibition of apoptotic progression. Environ Mol Mutagen. 2015;56(3):333-346. doi:10.1002/em.21919 
28. Park J, Ha MK, Yang N, Yoon TH. Flow cytometry-based quantification of cellular au nanoparticles. Anal Chem. 2017;89(4):2449-2456. doi:10.1021/acs.analchem.6b04418

29. Grigore ME, Biscu ER, Holban AM, Gestal MC, Grumezescu AM. Methods of synthesis, properties and biomedical applications of $\mathrm{CuO}$ nanoparticles. Pharmaceuticals. 2016;9(4):75. doi:10.3390/ph9040075

30. Suleiman M, Mousa M, Hussein A, Hammouti B, Hadda TB, Warad I. Copper (II)-oxide nanostructures: synthesis, characterizations and their applications-review. J Mater Environ Sci. 2013;4 (5):792-797.

31. Jing X, Park JH, Peters TM, Thorne PS. Toxicity of copper oxide nanoparticles in lung epithelial cells exposed at the air-liquid interface compared with in vivo assessment. Toxicol in Vitro. 2015;29 (3):502-511. doi:10.1016/j.tiv.2014.12.023

32. Niska K, Santos-Martinez MJ, Radomski MW, Inkielewicz-Stepniak I. $\mathrm{CuO}$ nanoparticles induce apoptosis by impairing the antioxidant defense and detoxification systems in the mouse hippocampal HT22 cell line: protective effect of crocetin. Toxicol in Vitro. 2015;29 (4):663-671. doi:10.1016/j.tiv.2015.02.004

33. Ko J-W, Shin N-R, Park J-W, et al. Copper oxide nanoparticles induce collagen deposition via TGF- $\beta 1 / \mathrm{Smad} 3$ signaling in human airway epithelial cells. Nanotoxicology. 2018;12(3):239-250. doi:10.1080/17435390.2018.1432778

34. Festa RA, Thiele DJ. Copper: an essential metal in biology. Curr Biol. 2011;21(21):R877-R883. doi:10.1016/j.cub.2011.09.040

35. Means RT. Nutritional Anemia: Scientific Principles, Clinical Practice, and Public Health. Cambridge University Press; 2019.

36. Wooton-Kee CR, Jain AK, Wagner M, et al. Elevated copper impairs hepatic nuclear receptor function in Wilson's disease. J Clin Invest. 2015;125(9):3449-3460. doi:10.1172/JCI78991

37. Quinn JF, Crane S, Harris C, Wadsworth TL. Copper in alzheimer's disease: too much or too little? Expert Rev Neurother. 2009;9 (5):631-637.

38. Wang D, Lin Z, Wang T, et al. Where does the toxicity of metal oxide nanoparticles come from: the nanoparticles, the ions, or a combination of both? J Hazard Mater. 2016;308:328-334. doi:10.1016/j.jhazmat.2016.01.066
39. Medici V, Sturniolo GC. Tetrathiomolybdate, a copper chelator for the treatment of Wilson disease, pulmonary fibrosis and other indications. IDrugs. 2008;11(8):592-606.

40. Pereira TC, Campos MM, Bogo MR. Copper toxicology, oxidative stress and inflammation using zebrafish as experimental model. J Appl Toxicol. 2016;36(7):876-885. doi:10.1002/jat.33 03

41. Rakshit A, Khatua K, Shanbhag V, Comba P, Datta A. $\mathrm{Cu}(2+)$ selective chelators relieve copper-induced oxidative stress in vivo. Chem Sci. 2018;9(41):7916-7930. doi:10.1039/C8SC04041A

42. Hotamisligil GS, Davis RJ. Cell signaling and stress responses. Cold Spring Harb Perspect Biol. 2016;8(10):a006072. doi:10.1101/cshperspect.a006072

43. Coulthard LR, White DE, Jones DL, McDermott MF, Burchill SA. p38MAPK: stress responses from molecular mechanisms to therapeutics. Trends Mol Med. 2009;15(8):369-379. doi:10.1016/j. molmed.2009.06.005

44. Park JW, Lee IC, Shin NR, et al. Copper oxide nanoparticles aggravate airway inflammation and mucus production in asthmatic mice via MAPK signaling. Nanotoxicology. 2016;10(4):445-452. doi:10.3109/ 17435390.2015.1078851

45. Lee IC, Ko JW, Park SH, et al. Copper nanoparticles induce early fibrotic changes in the liver via TGF-beta/Smad signaling and cause immunosuppressive effects in rats. Nanotoxicology. 2018;12 (6):637-651. doi:10.1080/17435390.2018.1472313

46. Raman M, Earnest S, Zhang K, Zhao Y, Cobb MH. TAO kinases mediate activation of $\mathrm{p} 38$ in response to DNA damage. EMBO J. 2007;26(8):2005-2014. doi:10.1038/sj.emboj.7601668

47. Wood CD, Thornton TM, Sabio G, Davis RA, Rincon M. Nuclear localization of p38 MAPK in response to DNA damage. Int J Biol Sci. 2009;5(5):428-437. doi:10.7150/ijbs.5.428

48. Yi C, Liu D, Fong CC, Zhang J, Yang M. Gold nanoparticles promote osteogenic differentiation of mesenchymal stem cells through p38 MAPK pathway. ACS Nano. 2010;4(11):6439-6448. doi:10.1021/nn101373r
International Journal of Nanomedicine

\section{Publish your work in this journal}

The International Journal of Nanomedicine is an international, peerreviewed journal focusing on the application of nanotechnology in diagnostics, therapeutics, and drug delivery systems throughout the biomedical field. This journal is indexed on PubMed Central, MedLine, CAS, SciSearch ${ }^{\mathbb{}}$, Current Contents ${ }^{\mathbb{R}} /$ Clinical Medicine, $^{-}$
Journal Citation Reports/Science Edition, EMBase, Scopus and the Elsevier Bibliographic databases. The manuscript management system is completely online and includes a very quick and fair peer-review system, which is all easy to use. Visit http://www.dovepress.com/ testimonials.php to read real quotes from published authors. 